\title{
Concentrated nanofat: a modified fat extraction promotes hair growth in mice via the stem cells and extracellular matrix components interaction
}

\author{
Zehua $\mathrm{Li}^{1,2 \# \wedge}$, Jinrong Zhang ${ }^{1,2 \#}$, Meng $\mathrm{Li}^{1}$, Lingzhi Tang ${ }^{1}$, Hongwei Liu ${ }^{1 \wedge}$ \\ ${ }^{1}$ Department of Plastic Surgery, the First Affiliated Hospital of Jinan University, Guangzhou, China; ${ }^{2}$ Innovative Technology Research Institute of \\ Tissue Repair and Regeneration, Key Laboratory of Regenerative Medicine, Ministry of Education, Guangzhou, China \\ Contributions: (I) Conception and design: Z Li, H Liu; (II) Administrative support: None; (III) Provision of study materials or patients: None; \\ (IV) Collection and assembly of data: Z Li, J Zhang, M Li; (V) Data analysis and interpretation: None; (VI) Manuscript writing: All authors; (VII) \\ Final approval of manuscript: All authors. \\ "These authors contributed equally to this work. \\ Correspondence to: Hongwei Liu. Department of Plastic Surgery, the First Affiliated Hospital of Jinan University, Guangzhou, China. \\ Email: liuhongwei0521@hotmail.com.
}

\begin{abstract}
Background: Fat graft transplantation seems a promising cell therapy for hair loss. However, impurities in lipoaspirate weaken the treatment effect. Here, we developed the lipoaspirate extraction method then investigate the effect and mechanism on hair growth-promoting in a mouse model.

Methods: Fat graft was prepared into concentrated nanofat (CNF), decellularized CNF (DCNF), and adipose-derived stem cells (ADSCs). They were injected subcutaneously in the back of depilated mice to test the hair promoting effect. Conditioned media (CM) from the adipose extracts were applied to dermal papilla cells (DPCs) to evaluate the cell viability and the anagen related signal.

Results: CNF and a high dose of ADSCs promoted hair growth and induced telogen-to-anagen transition in depilated mice. DCNF and a low dose of ADSCs did not show such effect; however, hair growth was promoted when they were used in combination. In vitro study showed the CNF-CM treated DPCs exhibited increased proliferation, migration, cell cycle progression, and elevated $\mathrm{Wnt} / \beta$-catenin pathway protein levels compared with the other treatment groups.

Conclusions: CNF has a better effect than ADSCs in hair promotion via activating the DPCs and anagen induction. In this nature complex of stem cells (SCs) and extracellular matrix (ECM), ECM serves a significant supplementary role and amplifies the power of ADSCs. These results supply a theoretical basis on the clinical application of $\mathrm{CNF}$ to treat hair loss.
\end{abstract}

Keywords: Hair loss; adipose-derived stem cells (ADSCs); extracellular matrix (ECM); cell therapy

Submitted Aug 05, 2020. Accepted for publication Sep 11, 2020.

doi: $10.21037 /$ atm-20-6086

View this article at: http://dx.doi.org/10.21037/atm-20-6086

\section{Introduction}

Hair loss is a common skin disorder. Although not life threatening, it has a significant effect on physical appearance, causing psychological stress. It also imposes an extra financial burden for people experiencing hair loss because of the cost of treatments, including nutritional shampoos, tonics, vitamin supplements, and medications that are routinely used to restore hair growth. Conventional treatments for hair loss include drugs and hair

^ ORCID: Zehua Li, 0000-0002-5624-5600; Hongwei Liu, 0000-0002-2591-8956. 
transplantation, which are also limited by drug resistance, adverse effects or insufficient graft sources (1). Therefore, an alternative treatment method suitable for clinical application is urgently needed.

Cell therapy, represented by mesenchymal stem cells (MSCs), has shown promising results in promoting hair growth. The adipose derived stem cells (ADSCs) induced telogen to anagen of hair follicles (HFs) in mice and elongation of in vitro organ cultured HFs. These effects can be enhanced by Ultraviolet B Preconditioning (2). Similar results were also achieved under the pretreatment of Minoxidil or cytokine $(3,4)$. Dermal papilla cells (DPCs) lost their traits after culturing. In Huang's study, a core-shell sphere of mixed ADSCs and DPCs restored and exhibited superior DP characteristics, which was demonstrated by hair inducing power in animal model (5). However, the extraction and cultivation of stem cells (SCs) introducing exogenous enzymes in the preparation process may cause contamination. Also, during large-scale cells cultivation of required for the therapeutic dosage, there are chances of SC differentiation and carcinogenesis. The time consuming, licensing and ethical issues also barrier their clinical application.

Transplantation of fat graft or its extract is regarded as a clinically feasible form of cell therapy for numbers of ADSCs inhabit in it $(6,7)$. However, as is known that fat grafts hold an extensive amount of tumescent fluid and oil, these components often cause inflammation, infection, poor graft survival, or absorption after transplantation $(8,9)$. Therefore, many efforts have been made to remove these invalid substances from lipoaspirates using physical methods, retaining the effective components such as ADSCs (10-12). In 2013, Tonnard et al. (13) introduced the concept of 'nanofat', in which the connective fibers were eliminated, and the fat graft was processed into an emulsion. This modification advanced the ADSCs-base cell therapy and fat transplantation; however, the ineffective components were not eliminated.

In many researches on cell therapy, the role of SCs is often paid more attention, while that of extracellular matrix $(\mathrm{ECM})$ is easily ignored. Since the subcomponents of fat grafts are ADSCs and ECM, some researchers have recognized that ECM plays a regulatory effect on SCs, and the SCs-ECM interaction seem to produce a synergistic biological effect.

In this study, we aimed to improve the nanofat processing for enhanced elimination of water and oil contaminants, to get a more concentrated nanofat (CNF). We then investigated the hair growth-promoting effect of the lipoaspirate extracts and potential underlying mechanisms in depilated C57BL/6J mice to explain whether the ECM or SCs are responsible for the therapeutic effect from the perspective of tissue engineering.

We present the following article in accordance with the ARRIVE reporting checklist (available at http://dx.doi. org/10.21037/atm-20-6086).

\section{Methods}

\section{Preparation of CNF and decellularized CNF (DCNF)}

Human lipoaspirates were harvested from six healthy women with no contraindications. A multiport 3-mm liposuction cannula with sharp side pores of diameter $1 \mathrm{~mm}$ (Tulip Medical Products, San Diego, CA, USA) was used for aspiration. The lipoaspirate mixtures were allowed to stand for 10 minutes, and then the separated upper portion of fat was transferred to centrifuge tubes for the first round of centrifugation ( $300 \mathrm{~g}, 3$ minutes), resulting in three separate layers. The bottom liquid layer was discarded. The middle fat layer and top oil layer were collected into syringes. This step removed a significant amount of water. According to the methods described by Tonnard et al., two 10-mL syringes were connected via a Luer-Lock connector, and the fat was shifted back and forth between the two syringes 30 times to produce the nanofat. The amount of nanofat in the connected syringes was adjusted to be almost similar, and negative pressure was simultaneously applied to both ends of the syringe several times (Figure 1A) to ensure that the emulsified fat with an initially uniform density becomes uneven. The fat was centrifuged again under the same conditions described above, resulting in its separation into three layers. The top oil layer and bottom water layer were removed, and the middle layer was kept as the desired product-the CNF. This step removed a significant amount of oil.

The CNF was collected in a syringe, maintained in a sterile condition, and frozen at $-80^{\circ} \mathrm{C}$ for 24 hours, followed by rewarming over a gradient of $-20^{\circ} \mathrm{C}$ for 12 hours, $4^{\circ} \mathrm{C}$ for 12 hours, and $37^{\circ} \mathrm{C}$ for 1 hour. This freeze-thaw cycle was performed three times, ensuring that all the cells in the sample were dead. The processed fat was then centrifuged again to remove the cell debris so that the oil accounting for one-fourth to one-third of the total volume was separated. The remaining adipose extract was the DCNF, which was stored at $4{ }^{\circ} \mathrm{C}$ for use in further experiments as the control sample to assess the effects of ECM. 
A
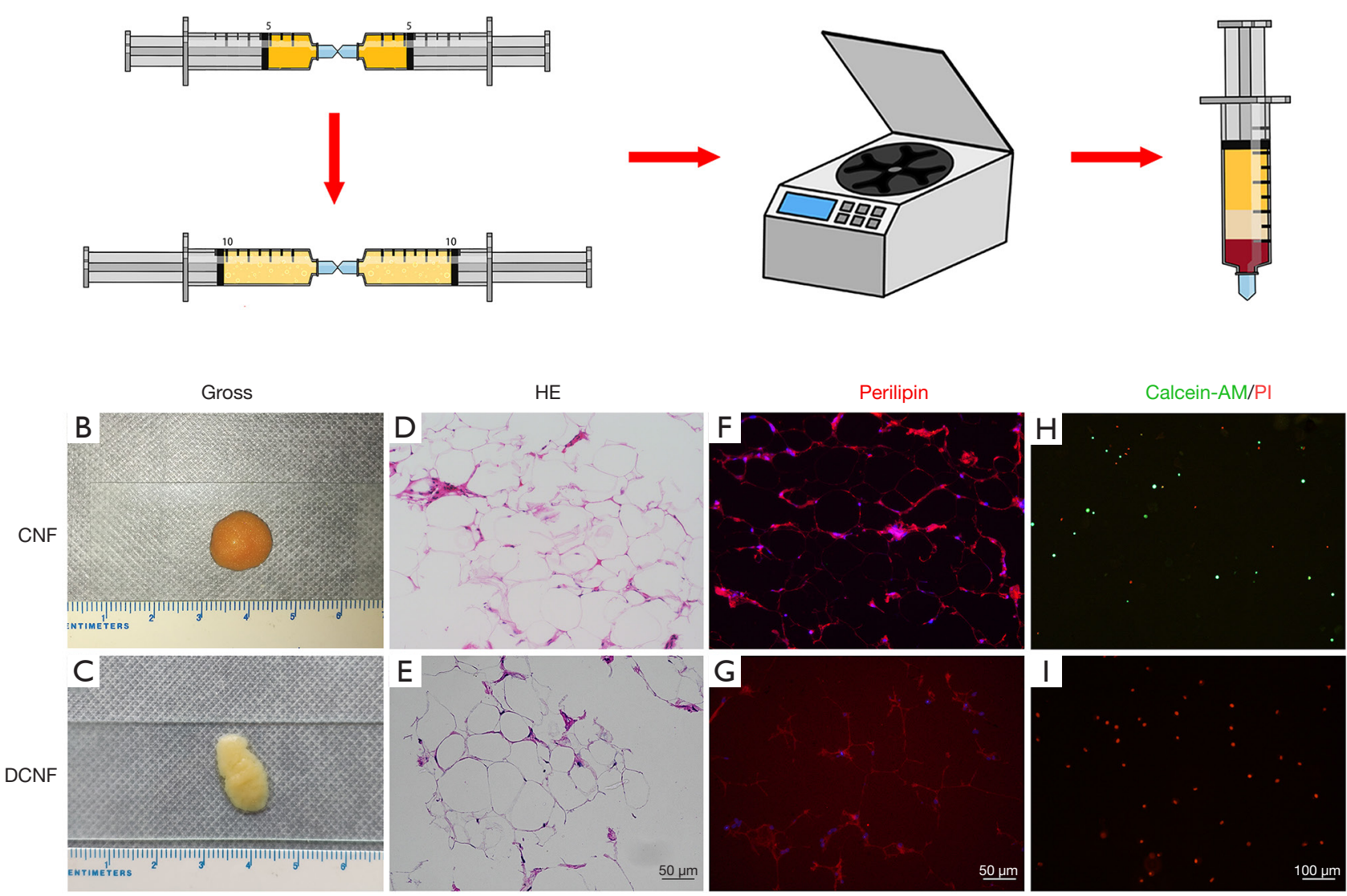

Figure 1 Characteristics and comparison of concentrated nanofat (CNF) and decellularized concentrated nanofat (DCNF). (A) Schematic diagram of CNF preparation; (B,C) general morphology of CNF and DCNF; (D,E) H\&E staining showing that CNF is denser than DCNF with smaller fat vesicles and more matrix components; $(\mathrm{F}, \mathrm{G})$ immunofluorescence of perilipin expression and localization in $\mathrm{CNF}$ and DCNF; (H,I) live/dead cell staining, proving green calcein AM-stained live cells and red propidium iodide-stained dead cells in the CNF, and only dead cells in DCNF.

\section{Stromal vascular fraction (SVF) isolation and fluorescence- activated cell sorting (FACS)}

CNF was digested with $0.2 \%$ collagenase, filtered, erythrocytes lysed, and centrifuged to prepare the SVF precipitates. After resuspending the SVF, half of the cell suspension was used for culture and amplification of ADSCs, and cells of the third to fifth generations were used in the later experiments. The other half of the SVF suspension was double-stained with calcein-AM (green, stains only live cells) and propidium iodide (PI; red, stains only dead cells). The numbers of living cells were counted by an automatic counter (CounterStar; Rui Yu Biotechnology Co., Ltd., Shanghai, China).

FACS quantified ADSC populations in the SVF on the positive expression of CD34, and negative expression of CD31 and CD45. SVFs were stained with monoclonal anti- human fluorochrome-conjugated antibodies, anti-CD31, anti-CD34, and anti-CD45 antibodies (BD Biosciences, San Jose, CA, USA) (14). On the surface marker expression profile, the numbers of ADSCs per unit CNF was quantified.

\section{Labeling of ADSCs}

The cells were transfected with a lentivirus carrying the plasmid pCDH-CMV-mKate2-2xNLS-T2A-Puro (Cytobiotech Co. Ltd., Xi'an, China) according to the manufacturer's protocol to track ADSCs in vivo. As a far-red fluorescent protein, higher brightness characterizes mKate2 and greater tissue penetration. It has, therefore, been widely used for labeling cells in vitro and real-time monitoring in vivo $(15,16)$. In brief, the ADSCs were seeded into 
24 -well plates $(20,000 /$ well) and grown to $30-50 \%$ confluence. The virus solution was added to the culture, which was incubated for 24-48 hours, and labeled cells were selected on plates holding $1 \mu \mathrm{g} / \mathrm{mL}$ puromycin.

\section{Animal model}

All animal experiments were performed with the approval of the ethical committee of the First Affiliated Hospital of Jinan University, and complied with the ARRIVE guidelines and carried out following the National Institutes of Health guide for the care and use of laboratory animals (NIH Publications No. 8023, revised 1978). Thirty sevenweek-old male C57BL/6J mice (18-22 g) were bought from the Experimental Animal Centre of Southern Medical University (Guangzhou, China). The mice were housed in a specific pathogen-free air-conditioned room $\left(25 \pm 2{ }^{\circ} \mathrm{C}\right.$ and $65 \% \pm 5 \%$ humidity) with a 12 -hour light/dark cycle. Their hair was in the telogen stage (17), and the dorsal area was waxed carefully without inducing skin damage to synchronize the hair follicle cycles.

The whole experiment consists of six groups and all animals were randomly assigned to each group. After intraperitoneal anesthesia by $4 \%$ chloral hydrate at $400 \mathrm{mg} / \mathrm{kg}, \mathrm{CNF}(0.1 \mathrm{~mL}), \mathrm{DCNF}(0.1 \mathrm{~mL})$, and PBS were subcutaneously injected into the left side of the dorsal skin. As numbers of ADSCs in CNF per unit had been calculated, ADSC suspensions equal to numbers of SCs in $0.1 \mathrm{~mL}$ of CNF were considered the low dose. Comparatively, those with 1,000 times the cell number were considered the high dose. Subsequently, ADSCs of low and high doses and DCNF $(0.1 \mathrm{~mL})$ in combination with low dose ADSCs were used respectively ( $\mathrm{n}=5 /$ group). The hair growth-promoting effects of each treatment were tested on hematoxylin and eosin (H\&E) tests, skin darkening, which showed the telogen-to-anagen conversion, and hair cycle progression. Photographs were captured at 8,12 , and 16 days after treatment (18). The mice treated with labeled ADSCs were also checked using the In-Vivo FX Pro Imaging system (Bruker, Madison, WI, USA) to determine the effects of SCs on hair growth.

\section{Histological analysis}

Fat (CNF and DCNF) and mouse skin tissues were embedded in paraffin, sectioned to $5 \mu \mathrm{m}$ thickness, and stained with H\&E. Immunofluorescence staining with primary antibodies targeting perilipin (ab3526, Abcam;
1:1,000) and Ki67 (ZM-0166, ZSGB-BIO, Beijing, China; 1:100), and then with secondary antibodies. Anagen (showed by darkened skin) hair follicles were counted in three highpower random fields under 100 magnification in all sections, and the mean value was calculated. The staining intensity was quantified as IOD/area using ImagePro Plus.

\section{Isolation and culture of dermal papilla cells}

DPCs were harvested from six 4-6-week-old male C57BL/6J mice. The vibrissa pads were cut, and follicles were isolated from the basal side of the mice under a stereomicroscope. The follicle capsules were pricked to expose the bases of the vibrissae follicles, and the dermal papillae were dissected using a thin needle. Isolated DPCs were placed in cell culture dishes holding the medium. After 1 week of culture, the cells reached confluence. Cells at passages 3-5 were used in the later experiments.

\section{Preparation of Conditioned Media (CMs)}

One milliliter of CNF or DCNF was added into sixwell plates. After that, $2 \mathrm{~mL}$ of serum-reduced medium (Dulbecco's modified Eagle medium with 2\% fetal bovine serum) was slowly added along the well walls to immerse the fat and was collected after 48 hours. Adipose tissue debris in the collected supernatant was first filtered using a 70- $\mu \mathrm{m}$ cell strainer (BD Falcon, USA), and then precipitated by centrifugation at 1,000 rpm for 3 minutes. The supernatant was removed and re-filtered using a $0.22-\mu \mathrm{m}$ syringe filter (Millipore, USA), and the acquired product was CNF-CM and DCNF-CM. The ADSC's suspension was adjusted to a concentration of $1.3 \times 10^{5}$ cells $/ \mathrm{mL}$. Then $1 \mathrm{~mL}$ ADSCs suspension with $1 \mathrm{~mL}$ medium was added to the six-well plate to support an equivalent amount of ADSCs and medium volume. When the cells reached $80 \%$ confluence, the medium was replaced with serum-reduced medium and cultured for a further 48 hours. The supernatant was centrifuged and filtered to retrieve the ADSCs-conditioned medium (AD-CM). The collected medium was mixed with the serum-reduced medium in a 1:1 volume ratio to prepare a 50\%-concentrated CMs (CNF-CM, DCNF-CM, and $\mathrm{AD}-\mathrm{CM})$ for the subsequent experiments.

\section{Cell viability, migration, and cell cycle analysis}

DPCs were treated with all three CMs, and the control group samples were treated with the serum-reduced 
medium. Cell viability was determined using the Cell Counting Kit-8 (Dojindo, Japan) according to the manufacturer's instructions, with the absorbance of the solution measured at $450 \mathrm{~nm}$.

Cell migration was analyzed using a wound-healing assay. After DPCs reached $90 \%$ confluence, a wound was created by scratching the cells with a $10-\mu \mathrm{L}$ pipette tip. The cells were treated with the CMs of the distinct groups, and the wound area was imaged with a reverse phase-contrast microscope (IX61 FL, Olympus, Japan) at 24-hour intervals. Quantitative analysis was performed using ImagePro Plus.

For the cell cycle analysis, the CMs-treated DPCs were harvested and fixed with $70 \%$ ethanol at $4{ }^{\circ} \mathrm{C}$ overnight. The cells were then washed twice and incubated in phosphatebuffered saline (PBS) holding $50 \mu \mathrm{g} / \mathrm{mL}$ RNase, followed by staining with $10 \mu \mathrm{g} / \mathrm{mL}$ PI. The stained samples were analyzed using a FACScan flow cytometer (BD Biosciences), and the DNA content was quantified using FlowJo $\mathrm{X}$ software (Tree Star, OR, USA).

\section{Western blotting}

Protein extracts were isolated from the CM-treated cells using Radio-Immunoprecipitation Assay (RIPA, Pierce, MA, USA) protein lysis buffer holding $1 \mathrm{mM}$ Phenylmethanesulfonyl fluoride (PMSF). The total protein was quantified using the bicinchoninic acid assay kit (Thermo Scientific, USA). Protein samples were separated by $10 \%$ sodium dodecyl sulfate-polyacrylamide gel electrophoresis and transferred onto polyvinylidene fluoride membranes. Following blocking, the membranes were incubated with primary $\beta$-catenin (sc-7963, Santa Cruz Biotechnology, USA; 1:1,000), Lef1 (sc-374412, Santa Cruz Biotechnology; 1:200), and cyclin D (Cat No. 2978, CST, USA; 1:1,000), and then with HRP-coupled secondary antibodies (Abcam; $1: 3,000)$ at room temperature for 1 hour. Immune complexes were detected using an enhanced chemiluminescence (ECL) substrate (TransGen Biotech, China), and the band intensity was analyzed using ImagePro Plus (19).

\section{Statistical analyses}

SPSS 20.0 software (SPSS Inc., Chicago, IL, USA) was used for all statistical analyses. All data are expressed as the mean \pm standard deviation of at least three independent experiments. Differences between two groups were assessed using Student's $t$-test. We assessed differences among multiple groups with a one-way analysis of variance.
Statistical significance was judged at $\mathrm{P}<0.05$.

\section{Results}

\section{Characteristics of CNF and DCNF}

The CNF had a smooth surface, with a uniform and fine texture, and aggregated quickly, resembling a water droplet when dropped onto a glass slide (Figure 1B). Following repeated freeze-thaw decellularization cycles, the CNF lost its ability to aggregate and become loose, showing a semimelted paraffin appearance (Figure 1C). The histological analysis revealed that the tissue structure of CNF was not damaged after decellularization, as both CNF and DCNF kept the original honeycomb structure. However, DCNF had more giant vesicles and fewer matrix components, which reduced its density; this was consistent with its general morphology (Figure 1D,E).

Immunofluorescence showed intense positive staining of perilipin in the CNF group, with weak staining in the DCNF group (Figure 1F,G). The SVF extracted from CNF contained both green calcein AM-positive (live) cells and red PI-positive (dead) cells, whereas the cells extracted from DCNF were exclusively stained with PI, and no adherent cells grew during culture (Figure 1H,I). These findings confirmed that the repeated freeze-thaw cycles removed all live-cell components from the CNF, which was initially enriched with ADSCs and left the ECM components with complete structure, which was considered the 'scaffold'.

\section{Quantification of ADSCs in CNF}

The total live cell number in the cell suspension was approximately $(3.7 \pm 0.4) \times 10^{5} / \mathrm{mL}$, determined using an automatic cell counter; the subsequent flow cytometry analysis for ADSC surface markers (CD34+, CD31-, and CD45-) showed that the average proportion of ADSCs was $34.4 \% \pm 8.1 \%$ (Figure $2 A$ ). Therefore, we considered the average number of ADSCs in $1 \mathrm{~mL}$ of CNF to be $1.3 \times 10^{5}$. ADSCs successfully transfected with the mKate2-encoding lentivirus showed red fluorescence in the nucleus under a fluorescence microscope, achieving a transfection positivity rate of over $90 \%$ after antibiotic screening (Figure $2 B$ ).

\section{CNF promoted hair growth but was ineffective after decellularization}

No animals were infected or dead in our study. First, we studied the effect of CNF on hair growth. We tried 
A

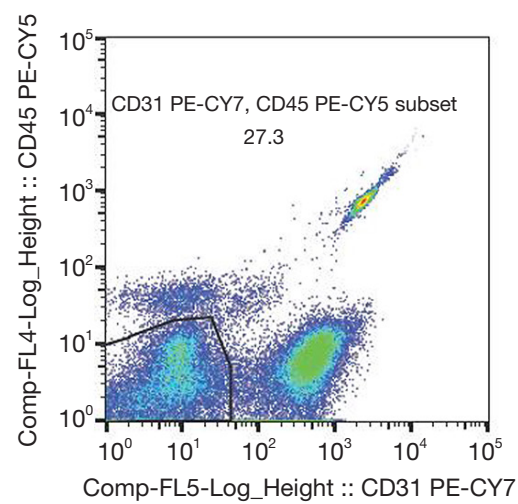

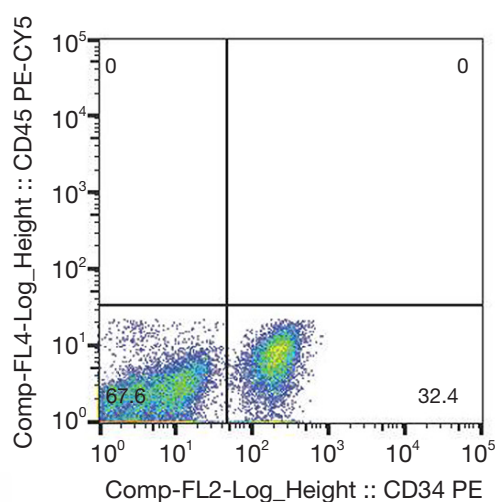

B

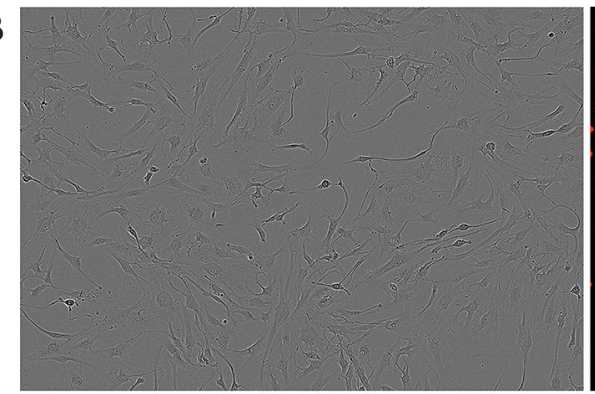

bright light

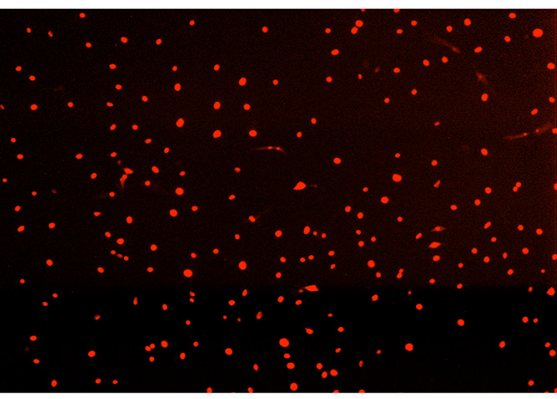

mkate2

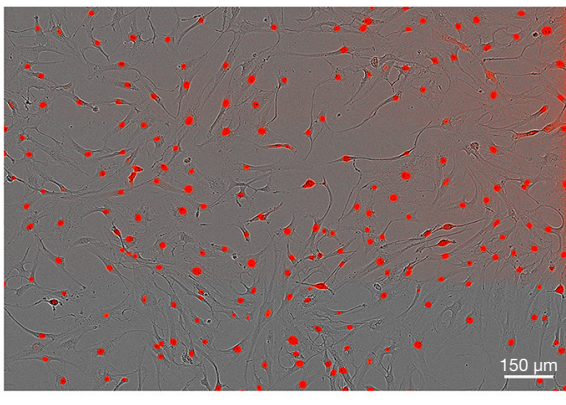

merge

Figure 2 Screening and labeling of adipose-derived stem cells (ADSCs). (A) Flow cytometry analysis of the stromal vascular fraction extracted from the $\mathrm{CNF}$; CD34 ${ }^{+} \mathrm{CD} 31^{-} \mathrm{CD} 45^{-}$cells were considered ADSCs; (B) human ADSCs were transfected with lentivirus expressing mKate2; the red fluorescence was expressed in the cell nuclei.

different doses and concluded that $0.1 \mathrm{~mL}$ was the lowest effective dose. In the CNF-treated mice (5/5), it was faster than that in the DCNF- or PBS-treated mice (5/5). The area of the CNF-treated skin changed from pink to dark black on day eight on an average, showing that HFs were in the growth phase, whereas the area of the DCNF- and PBS-treated skin showed only a slight color change. On day 12 , hair grew out of the skin surface at the CNF injection site, and the color of the depilated area gradually deepened with the decrease in distance from the injection site. On day 16 , hair was observed growing beyond the body surface in all the groups; however, the hair in the CNF group was thicker, and hair in the other two groups grew later with a relatively reduced length and density (Figure $3 A$ ).

$\mathrm{H} \& \mathrm{E}$ staining on day 12 after treatment confirmed that the HFs in the CNF group showed a higher number and density of anagen HFs. Morphologically, the HFs and papillae were larger in volume, reaching deeper into the fat layer and the superficial surface of the muscle fascia, with hair shafts penetrating the epidermis. However, the size of the dermal papillae was insignificant in the other two groups. There were still many follicles in the early anagen phase, with their hair fibers not protruding out of the skin surface (Figure 3B). The CNF group had the highest number of HFs among the three groups $(\mathrm{P}=0.026, \mathrm{CNF} v s$. DCNF; $\mathrm{P}=0.024, \mathrm{CNF} v s . \mathrm{PBS} ; \mathrm{P}=0.561$, DCNF vs. PBS; Figure 3C). Immunofluorescence staining for Ki67, a marker of cell proliferation, showed proliferating cells in the hair matrix and dermal papilla compartments (Figure 3D), with the highest density of proliferating cells observed in the CNF-treated group $(\mathrm{P}<0.05$; Figure $3 E)$.

\section{High dose ADSCs showed hair growth-promoting effects}

We also used two different doses to verify the efficacy of ADSCs used alone as numbers of ADSCs corresponding to $0.1 \mathrm{~mL}$ of $\mathrm{CNF}$ was only about $1.3 \times 10^{4}$, we defined this amount as the low dose, which was not sufficient for cell therapy. Besides, we used 1,000 times this amount as the high dose. It was proved that a high dose of ADSCs showed hair growth-promoting effect, as evidenced by the darker pigmented skin at the injection site, which indicated the initial anagen phase of the affected HFs (Figure 4A). However, treatment with the low dose of 

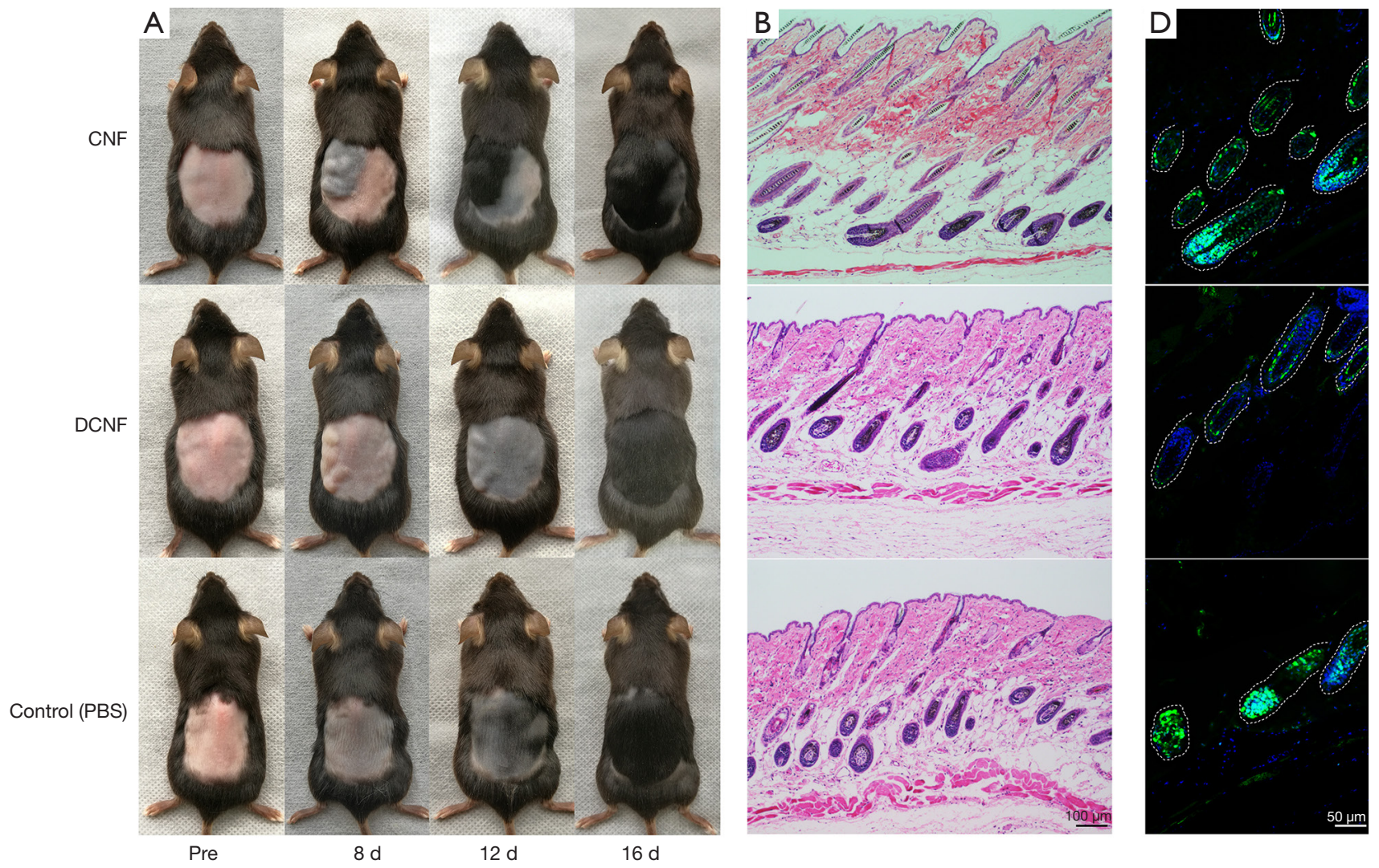

C

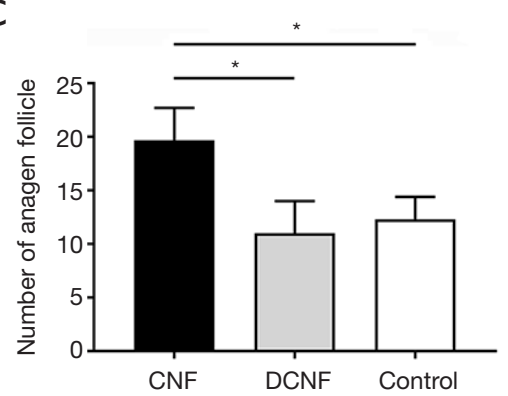

E

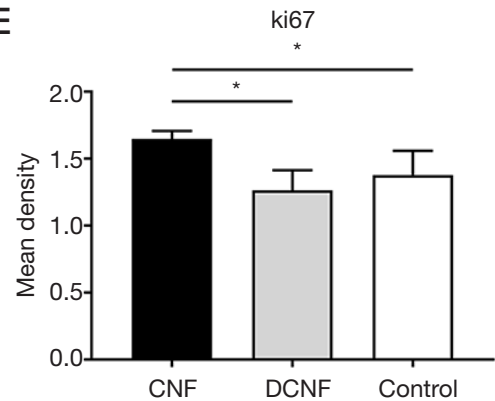

Figure 3 Concentrated nanofat (CNF) promotes hair growth and accelerates telogen-to-anagen transition in C56BL/6 mice. (A) After hair depilation, the mouse dorsal skin was treated with CNF, decellularized CNF (DCNF), and phosphate-buffered saline (PBS; blank control). Photographs were taken at 8,12, and 16 days after treatment. Skin pigmentation and hair shafts growing out of the body were observed earlier in the CNF-treated group. (B) H\&E staining of the dorsal skin from all groups on day 12. (C) Quantification of anagen hair follicles. (D) Immunofluorescence of Ki67 expression on day 12. The positive cells (green) with DAPI (blue) counterstaining were in the hair matrix and dermal papilla compartments. (E) Quantification of Ki67. Data are shown as mean \pm SD. ${ }^{*}, \mathrm{P}<0.05$.

ADSCs did not exhibit a hair growth-promoting effect, as no difference in color between the injection site and surrounding areas, similar to the negative control (DCNF) and blank (PBS) groups (Figure 4B). In vivo imaging showed fluorescence signals on the back of mice treated with high dose ADSCs, and the fluorescent range was consistent with the injection site or the skin hyperpigmentation area (Figure 4C). However, low dose ADSCs injection did not result in any visible fluorescent signals (Figure 4D). 

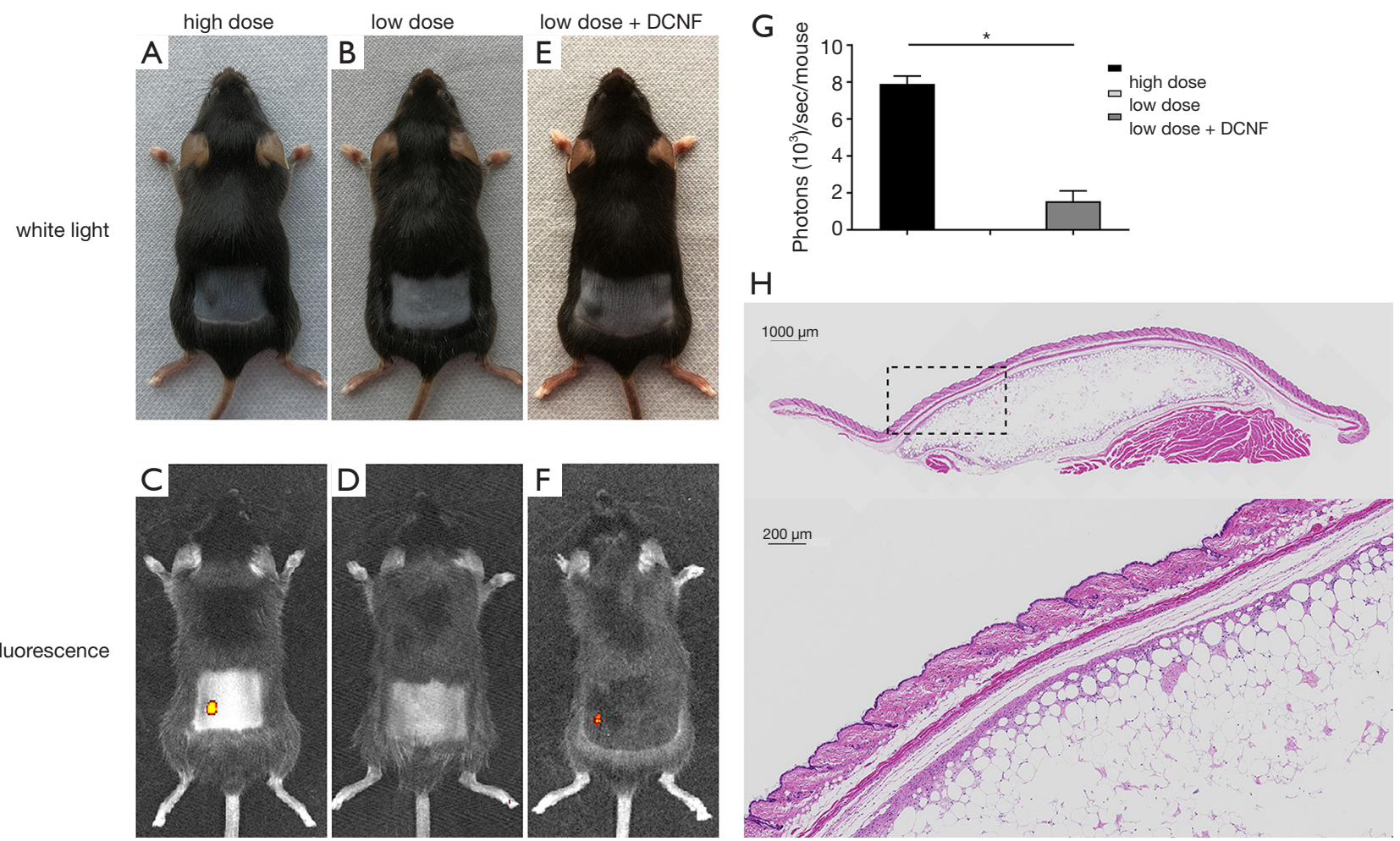

Figure 4 Effects of different doses of adipose-derived stem cells (ADSCs) and the combination of ADSCs and decellularized concentrated nanofat (DCNF) on hair growth. (A,B) After depilation, the dorsal skins of C56BL/6 mice were treated with ADSCs at high dose and low dose. Skin pigmentation and growing hair shafts were checked. (C,D) In vivo fluorescence images following injection with mKate2-labeled ADSCs (high and low doses). The ranges of fluorescence signals were consistent with skin hyperpigmentation and hair growth. (E,F) The combination of DCNF+ADSCs (low dose) on depilated skin displayed accelerated hair growth and weak fluorescent signals at the injection site. (G) Quantitative analysis of mKate2 signal intensity expressed as photons/s/mouse. (H) H\&E staining of the transplanted CNF under the dorsal skin at three months post-transplantation. Data are shown as mean $\pm \mathrm{SD}$. ${ }^{*}, \mathrm{P}<0.05$.

\section{The hair growth-promoting effect of DCNF was restored following its combination with ADSCs}

Low dose ADSCs alone were ineffective as described above. However, when combined with DCNF, deeper hyperpigmentation was observed at the injection site where the hair growth accelerated and the anagen advanced (Figure 4E). Further evidence of the combination was obtained by in vivo imaging analysis, in which even weak fluorescence could only be detected in the DCNF + ADSCs group despite administration of the same low dose of cells (Figure 4F). Quantitative analysis of in vivo fluorescence signals, which illustrates the difference of fluorescence intensity in each group (Figure $4 G$ ).

\section{Histological changes of CNF before and after transplantation}

Three months after transplantation, most CNF graft survived normally with only sporadic macrophages in it. Histological tests showed that internal structure of the post-transplanted CNF was similar to that of the pretransplanted CNF. The transplanted CNF was encapsulated in capsule, presenting a honeycomb-like composition of adipocytes and connective tissues. Most cells in the outer edge were preadipocytes holding small lipid droplets. On the inner side, the primary cells gradually transitioned to mature adipocytes with considerable lipid drops. In the center, there were diffused blank areas formed because of poor tissue survival (Figure 4H).

\section{CNF-CM promoted the proliferation and migration of DPCs and accelerated the cell cycle}

DPCs treated with CNF-CM proliferated faster than those in the other CM-treated groups (Figure 5A). The wound- 
A
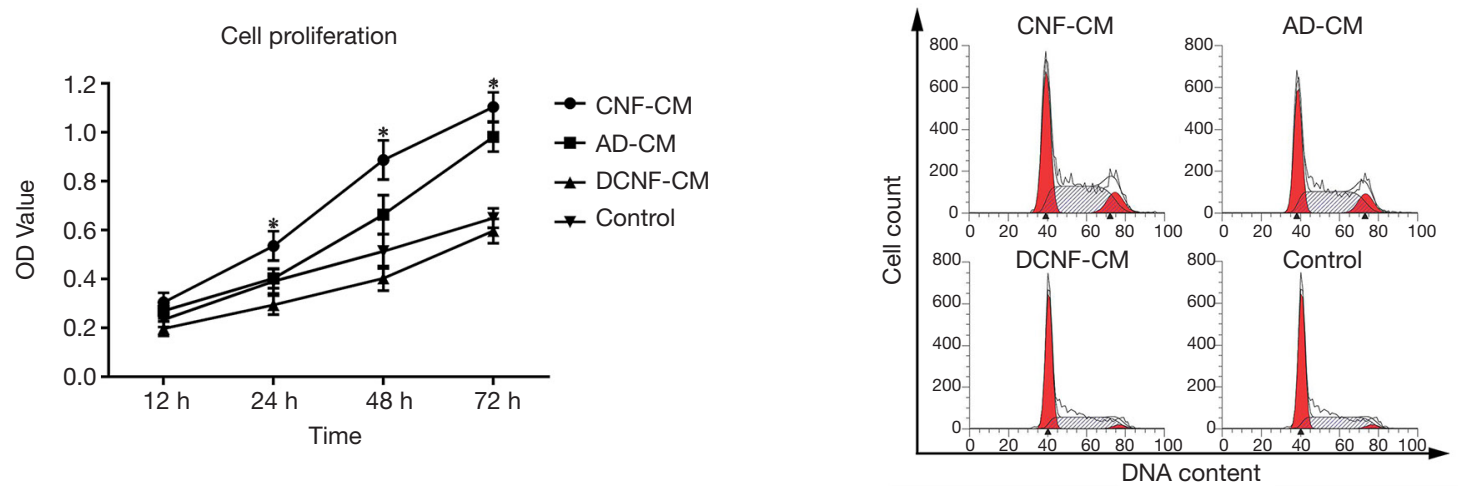

B

CNF-CM

$\mathrm{AD}-\mathrm{CM}$

DCNF-CM

Control

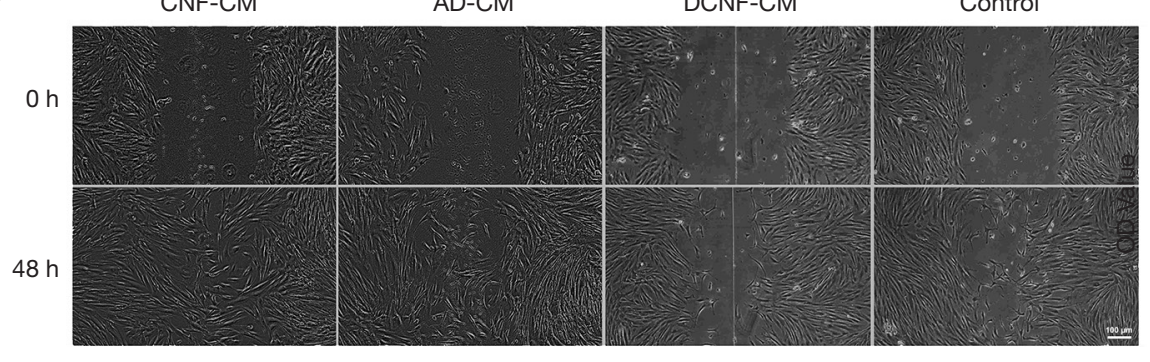

E
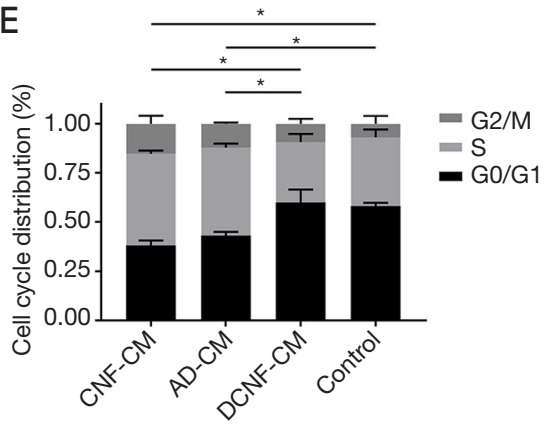

C

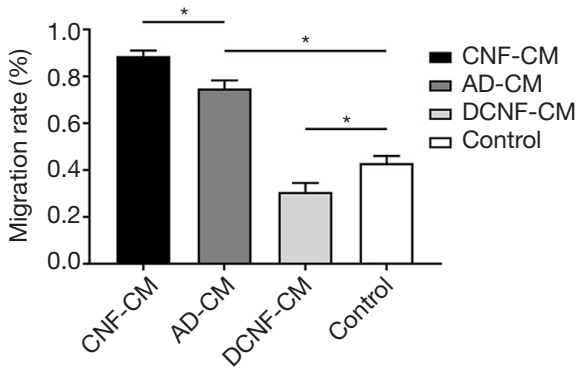

$\mathrm{F}$

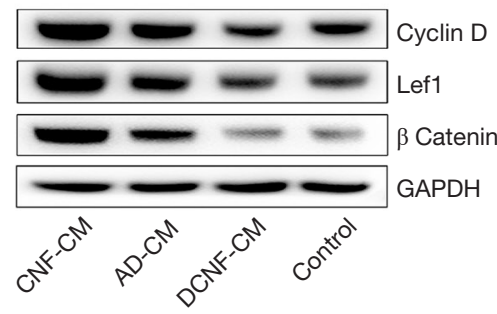

G

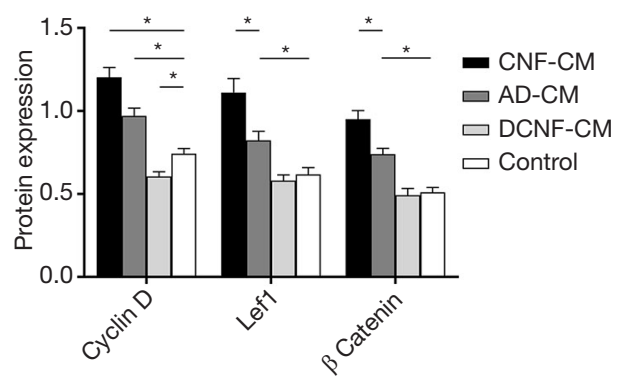

Figure 5 Culture of dermal papilla cells (DPCs) treated with the conditioned media (CM) of concentrated nanofat (CNF), decellularized CNF (DCNF), and adipose-derived stem cells (ADSCs). (A) Cell proliferation was measured using the Cell Counting Kit-8 method, and a cell proliferation curve was drawn; (B,C) wound-healing assay; (D,E) flow cytometry profile of the cell cycle; (F,G) Western blotting for the quantification of $\beta$-catenin, Lef1, and cyclin D protein expression in DPCs. CNF-CM significantly increased the expression of these proteins. Data are shown as mean $\pm \mathrm{SD}$. *, $\mathrm{P}<0.05$.

healing assay showed the same trend. CNF-CM or AD$\mathrm{CM}$ enhanced the migration of DPCs compared to that in the control group, and the migration in the DCNF$\mathrm{CM}$ group was slower. DPCs cultured in CNF-CM almost wholly covered the areas in the scratch wound within 48 hours, whereas DPCs in the other groups showed limited migration with incomplete coverage of the scratched areas during the same period (Figure 5B,C). The cell cycle analysis showed that treatment with CNF-CM increased the proportion of S-phase cells by 1.5 and 1.3 times compared to that in the DCNF-CM and control group, respectively.
However, there was no significant difference between the CNF-CM and AD-CM groups (Figure 5D,E).

Western blot detected the signature proteins associated with anagen induction to investigate the signaling mechanism underlying the HF development in different treatment groups. DPCs treated with CNF-CM or AD-CM showed higher expression of $\beta$-catenin, Lef1, and cyclin D than the normal control. DCNF-CM treated group showed lower expression (Figure 5F,G). These results suggested that CMs holding cell paracrine substance-induced DPCs anagen activity through the Wnt pathway, and because of 
extracellular matrix enhances SC paracrine function, $\mathrm{CNF}$ has a better effect.

\section{Discussion}

Fat transplantation has been performed for over 100 years but has long been associated with adverse reactions (20). In 2015, a study proposed that removing oil and water before transplantation improved the survival of transplanted fat and reduced the incidence of adverse reactions (21), and this is now widely accepted. In the present study, we changed the lipoaspirate extraction procedure to further remove the ineffective components in it. Specifically, the nanofat, an intermediate product, was subjected to a negative pressure process using a syringe, followed by centrifugation to separate the components and remove the oil and water. This method was inspired by the principle of negative pressure cavitation extraction technology, widely used in pharmaceutical, food, and phytochemistry industries $(22,23)$. Although a simple process, this was essential to separate the emulsified nanofat; under the negative pressure of the syringe, many cavities were formed in the nanofat, resulting in an uneven distribution that facilitated the separation of substances with unique properties by centrifugation. The CNF was thus successfully retrieved, showing over a 100-fold higher amount of ADSCs than the original nanofat sample (13).

This practical and straightforward preparation method of CNF is helpful for clinical application. As is known, patients still need to face the potential post-surgical risks of fat liquefaction, necrosis, and infection. Therefore, an in vitro lipoaspirate processing method should be as simplified, quick, and sterile as possible. No additives were used in our process to minimize the chances of infection. Our entire processing time was $10-15$ minutes, involving only the push-pull action of a syringe and two rounds of centrifugation. As a simplified description of this process, we refer to this method as the 'Triple Three' rule: 3 minutes of centrifugation, 30 fragmentations, and $300 \mathrm{~g}$ centrifugal force. These parameters are easy for operators to remember.

For the decellularization of CNF, we used freezethaw cycles, the most common physical decellularization technique $(24,25)$. A single freeze-thaw cycle is used to reduce the immune response caused by leukocyte infiltration in the ECM (26), whereas multiple freeze-thaw cycles can remove cells while retaining the matrix structure $(27,28)$. After our freeze-thaw circulation processing, DCNF showed negative perilipin expression and only dead cells in the live-dead cell staining, which indirectly proved the living cells in CNF destroyed and the bioactive proteins degraded, leaving a structure-preserving ECM. Although the physical decellularization method alone cannot remove components including cell debris or nucleic acids, it allows a quick 'subtraction' process while preserving the original spatial structure of ECM without inducing exogenous substances.

SCs have a critical role in the maintenance and regeneration of tissues, and they were found in a specialized microenvironment, known as the niche. ECM is the vital composition of the SC niche and serves as a protective and supportive regulator. SCs are in contact with ECM, and their constant interaction directs the cell behavior for tissue regeneration (29). In the animal model used in our study, synchronized anagen of hair coat was induced after depilation (17) to compare the effects of the injected adipose extracts and an interval of two days was used to observe the dynamic changes of anagen hair growth (18). The in vivo experiment has shown that CNF could promote hair growth. Then we evaluated the effect of its subcomponents of SCs and ECM. As its decellularized product, the DCNF alone did not result in hair promotion, which showed that ADSCs in the CNF was responsible for the therapeutic effects, but not the ECM.

Further analysis of ADSCs showed that the number of cells used was a positive correlation with the hair promoting effect. Beyond that, two individually ineffective ingredients regained the function when used in combination. The function of ADSCs was amplified in ECM. These results further supported the basic principle of 'seed cells + scaffolds' in tissue engineering (30) and added new evidence to the cell therapy to prove that $\mathrm{CNF}$ is an ideal alternative for the SCs and ECM are working in combination, leading to an additive effect (31).

We also want to know the outcomes and changes of CNF before and after transplantation. As the histological results showed, the honeycomb-like structure was kept in most areas of the graft three months post-transplantation. Compared with the first graft, there were tiny preadipocytes in the periphery part under the capsule. On the inner side, the mature adipocytes gradually became dominant. We hypothesized that in the central zone of transplanted $\mathrm{CNF}$, adipose tissues had poor survival because of failing to establish blood supply in time, and ADSCs could not undergo adipogenesis. However, ADSCs in the periphery survived on the supply of adjacent host tissues and further developed into preadipocytes and adipocytes. They induced 
adipogenesis and replaced the poorly survived adipocytes in the center by inward migration (32), thus constantly exerting their effects on hair growth.

The paracrine manner is the dominant mechanism of ADSCs for cell therapy (33). CMs of SCs contain many self-released cytokines, which are used to investigate their paracrine function (34). In our findings, DPCs under CNF$\mathrm{CM}$ and $\mathrm{AD}-\mathrm{CM}$ treatment showed increased proliferation, accelerated cell cycle, and un-regulated Wnt/ $\beta$-Catenin expression. With the support of the ECM niche, ADSCs exhibited more potent paracrine effects, just as CNF did. Not surprisingly, the DCNF-CM treated DPCs were invalid, for it had no SCs that acted as the key element. From these facts, it was concluded that ECM perform an important supporting role. A combination of the SCs and ECM complex are more powerful than SCs alone.

A series of signals have been proved implicated in HF development, including Wnt, BMP, Shh, notch (35). Wnt initiates the HF neogenesis and development. BMPs also act in HF morphogenesis and tend to be dominant negative regulators. SHH plays an important role in HF regeneration. Notch participates in hair maturation. Among the known molecules involved in HF development, Wnt/ $\beta$-catenin pathway appears to be the most critical (36). In Demeng's study, Wnt initiated HF placodes formation. Forced activation of $\mathrm{Wnt} / \beta$-catenin led to the formation of thickened dermis and prematurely enlarged HFs (37). Blocking of the Wnt/ $\beta$-catenin by Dickkop 1 (DKK-1) demonstrated a decreased HF length and anagen-tocatagen transition (38). DPCs are believed to direct the HFs morphogenesis and precisely regulate their cycling (39). Studies report that the cytokines or biological agents treated DPCs induced hair growth, which has always been related with the activation of Wnt (40). Application of 3-Deoxysappanchalcone on C57BL/6 mice and DPCs resulted in anagen transition, hair growth and cell proliferation (41). In another study, DPCs from patients with and rogenetic alopecia abrogated hair differention ability. However, Wnt activation restored the ability of androgen-treated DPCs to induce differentiation (36). It was also found that the Wnt activatorstimulated DP spheroids reconstituted skin with enriched HFs (42). Our results are in agreement with such evidences that the $W n t / \beta$-Catenin signal responded in the CNF-CM and $\mathrm{AD}-\mathrm{CM}$ treated DPCs, which was characterized by the related molecules. In the absence of $\mathrm{Wnt}$, the cytoplasm $\beta$-Catenin is degraded by phosphorylation. With Wnt activation, $\beta$-catenin would no longer be phosphorylated and accumulate to form nuclear complexes with TCF/LEF factors, which activates the transcription of target genes and results in the biological effects (43). Therefore, we have reason to infer that the canonical $W n t / \beta$-Catenin may be one of the important pathways for DPCs to be activated by CNF.

Although we've found the beneficial effect of $\mathrm{CNF}$ on hair growth, there're still existed some limitations. Firstly, the component differences in the CM can be further analyzed by protein array. In addition, other signals may also be involved in hair morphogenesis and their joint effects should next be tested.

\section{Conclusions}

Our research developed a method for refining nanofat into a concentrated form named $\mathrm{CNF}$, regarded as nature SCs-ECM complex, which is able to induce anagen and promote hair growth by activating the DPCs via canonical Wnt/ $\beta$-catenin pathway expectedly. As the critical element of CNF, ADSCs manage the CNF biofunction, and ECM amplifies their power. Because of the advantages of high ADSCs concentration and available for immediate use in the clinic, CNF supplies a clinical approach of cell therapy for treating hair loss. Our study lays a theoretical foundation for the clinical application of adipose extracts.

\section{Acknowledgments}

Funding: This work was supported by the National Natural Science Foundation of China (No. 81372065, 81272100, and 81871563); Medical Scientific Research Foundation of Guangdong Province, China (A2016568).

\section{Footnote}

Reporting Checklist: The authors have completed the ARRIVE reporting checklist. Available at http://dx.doi. org/10.21037/atm-20-6086

Data Sharing Statement: Available at http://dx.doi. org/10.21037/atm-20-6086

Conflicts of Interest: All authors have completed the ICMJE uniform disclosure form (available at http://dx.doi. org/10.21037/atm-20-6086). The authors have no conflicts of interest to declare. 
Ethical Statement: The authors are accountable for all aspects of the work in ensuring that questions related to the accuracy or integrity of any part of the work are appropriately investigated and resolved. All animal experiments complied with the ARRIVE guidelines and carried out following the National Institutes of Health guide for the care and use of laboratory animals (NIH Publications No. 8023, revised 1978). The ethical committee approved this study of the First Affiliated Hospital of Jinan University.

Open Access Statement: This is an Open Access article distributed in accordance with the Creative Commons Attribution-NonCommercial-NoDerivs 4.0 International License (CC BY-NC-ND 4.0), which permits the noncommercial replication and distribution of the article with the strict proviso that no changes or edits are made and the original work is properly cited (including links to both the formal publication through the relevant DOI and the license). See: https://creativecommons.org/licenses/by-nc-nd/4.0/.

\section{References}

1. Gentile P, Garcovich S. Advances in Regenerative Stem Cell Therapy in Androgenic Alopecia and Hair Loss: Wnt pathway, Growth-Factor, and Mesenchymal Stem Cell Signaling Impact Analysis on Cell Growth and Hair Follicle Development. Cells 2019;8:466.

2. Jeong YM, Sung YK, Kim WK, et al. Ultraviolet B preconditioning enhances the hair growth-promoting effects of adipose-derived stem cells via generation of reactive oxygen species. Stem Cells Dev 2013;22:158-68.

3. Minoxidil Promotes Hair Growth through Stimulation of Growth Factor Release from Adipose-Derived Stem Cells

4. Choi N, Kim WS, Oh SH, et al. HB-EGF Improves the Hair Regenerative Potential of Adipose-Derived Stem Cells via ROS Generation and Hck Phosphorylation. Int J Mol Sci 2019;21:122.

5. Huang CF, Chang YJ, Hsueh YY, et al. Assembling Composite Dermal Papilla Spheres with Adipose-derived Stem Cells to Enhance Hair Follicle Induction. Sci Rep 2016;6:26436.

6. Perez-Meza D, Ziering C, Sforza M, et al. Hair follicle growth by stromal vascular fraction-enhanced adipose transplantation in baldness. Stem Cells Cloning 2017;10:1-10.

7. Anderi R, Makdissy N, Azar A, et al. Cellular therapy with human autologous adipose-derived adult cells of stromal vascular fraction for alopecia areata. Stem Cell Res Ther 2018;9:141.

8. Kang D, Luan J. Fat Necrosis After Autologous Fat Transfer (AFT) to Breast: Comparison of Low-Speed Centrifugation with Sedimentation. Aesthetic Plast Surg 2018;42:1457-64.

9. Mineda K, Kuno S, Kato H, et al. Chronic inflammation and progressive calcification as a result of fat necrosis: the worst outcome in fat grafting. Plast Reconstr Surg 2014;133:1064-72.

10. Condé-Green A, Wu I, Graham I, et al. Comparison of 3 techniques of fat grafting and cell-supplemented lipotransfer in athymic rats: a pilot study. Aesthet Surg J 2013;33:713-21.

11. Khater R, Atanassova P, Anastassov Y, et al. Clinical and experimental study of autologous fat grafting after processing by centrifugation and serum lavage. Aesthetic Plast Surg 2009;33:37-43.

12. Ansorge H, Garza JR, McCormack MC, et al. Autologous fat processing via the Revolve system: quality and quantity of fat retention evaluated in an animal model. Aesthet Surg J 2014;34:438-47.

13. Tonnard P, Verpaele A, Peeters G, et al. Nanofat grafting: basic research and clinical applications. Plast Reconstr Surg 2013;132:1017-26.

14. Philips BJ, Grahovac TL, Valentin JE, et al. Prevalence of endogenous CD34+ adipose stem cells predicts human fat graft retention in a xenograft model. Plast Reconstr Surg 2013;132:845-58.

15. Xie P, Hu X, Li D, et al. Bioluminescence Imaging of Transplanted Mesenchymal Stem Cells by Overexpression of Hepatocyte Nuclear Factor4a: Tracking Biodistribution and Survival. Mol Imaging Biol 2019;21:44-53.

16. Vuletic I, Liu J, Wu H, et al. Establishment of an mKate2-Expressing Cell Line for Non-Invasive RealTime Breast Cancer In Vivo Imaging. Mol Imaging Biol 2015;17:811-8.

17. Müller-Röver S, Handjiski B, van der Veen C, et al. A comprehensive guide for the accurate classification of murine hair follicles in distinct hair cycle stages. J Invest Dermatol 2001;117:3-15.

18. Enshell-Seijffers D, Lindon C, Kashiwagi M, et al. beta-catenin activity in the dermal papilla regulates morphogenesis and regeneration of hair. Dev Cell 2010;18:633-42.

19. Li Z, Li S, Li K, Jiang X, Zhang J, Liu H. A highly simulated scar model developed by grafting human thin split-thickness skin on back of nude mouse: The 
remodeling process, histological characteristics of scars. Biochem Biophys Res Commun 2020;526:744-50.

20. Simonacci F, Bertozzi N, Grieco MP, et al. From liposuction to adipose-derived stem cells: indications and technique. Acta Biomed 2019;90:197-208.

21. Qiu L, Su Y, Zhang D, et al. Identification of the Centrifuged Lipoaspirate Fractions Suitable for Postgrafting Survival. Plast Reconstr Surg 2016;137:67e-76e.

22. Misra NN, Koubaa M, Roohinejad S, et al. Landmarks in the historical development of twenty first century food processing technologies. Food Res Int 2017;97:318-39.

23. Luo M, Yang LQ, Yao XH, et al. Optimization of enzymeassisted negative pressure cavitation extraction of five main indole alkaloids from Catharanthus roseus leaves and its pilot-scale application. Sep Purif Technol 2014;125:66-73.

24. Crapo PM, Gilbert TW, Badylak SF. An overview of tissue and whole organ decellularization processes. Biomaterials 2011;32:3233-43.

25. Lu H, Hoshiba T, Kawazoe N, et al. Comparison of decellularization techniques for preparation of extracellular matrix scaffolds derived from three-dimensional cell culture. J Biomed Mater Res A 2012;100:2507-16.

26. Lehr EJ, Rayat GR, Chiu B, et al. Decellularization reduces immunogenicity of sheep pulmonary artery vascular patches. J Thorac Cardiovasc Surg 2011;141:1056-62.

27. Nonaka PN, Campillo N, Uriarte JJ, et al. Effects of freezing/thawing on the mechanical properties of decellularized lungs. J Biomed Mater Res A 2014;102:413-9.

28. Flynn LE. The use of decellularized adipose tissue to provide an inductive microenvironment for the adipogenic differentiation of human adipose-derived stem cells. Biomaterials 2010;31:4715-24.

29. Gattazzo F, Urciuolo A, Bonaldo P. Extracellular matrix: a dynamic microenvironment for stem cell niche. Biochim Biophys Acta 2014;1840:2506-19.

30. Murphy CM, O'Brien FJ, Little DG, et al. Cell-scaffold interactions in the bone tissue engineering triad. Eur Cell Mater 2013;26:120-32.

31. Sreejit P, Verma RS. Natural ECM as biomaterial for scaffold based cardiac regeneration using adult bone marrow derived stem cells. Stem Cell Rev Rep 2013;9:158-71.

32. Gregoire FM, Smas CM, Sul HS. Understanding adipocyte differentiation. Physiol Rev 1998;78:783-809.
33. Nauta A, Seidel C, Deveza L, et al. Adipose-derived stromal cells overexpressing vascular endothelial growth factor accelerate mouse excisional wound healing. Mol Ther 2013;21:445-55.

34. Pawitan JA. Prospect of stem cell conditioned medium in regenerative medicine. Biomed Res Int 2014;2014:965849.

35. Wang X, Tredget EE, Wu Y. Dynamic signals for hair follicle development and regeneration. Stem Cells Dev 2012;21:7-18.

36. Leirós GJ, Attorresi AI, Balañá ME. Hair follicle stem cell differentiation is inhibited through cross-talk between Wnt/ $\beta$-catenin and androgen signalling in dermal papilla cells from patients with and rogenetic alopecia. $\mathrm{Br} \mathrm{J}$ Dermatol 2012;166:1035-42.

37. Chen D, Jarrell A, Guo C, et al. Dermal $\beta$-catenin activity in response to epidermal Wnt ligands is required for fibroblast proliferation and hair follicle initiation. Development 2012;139:1522-33.

38. Kwack MH, Kim MK, Kim JC, et al. Dickkopf 1 promotes regression of hair follicles. J Invest Dermatol 2012;132:1554-60.

39. Topouzi H, Logan NJ, Williams G, et al. Methods for the isolation and 3D culture of dermal papilla cells from human hair follicles. Exp Dermatol 2017;26:491-6.

40. Bak DH, Lee E, Lee BC, et al. Boehmite enhances hair follicle growth via stimulation of dermal papilla cells by upregulating $\beta$-catenin signalling. Exp Dermatol 2020;29:341-8.

41. Kim YE, Choi HC, Lee IC, et al. 3-Deoxysappanchalcone Promotes Proliferation of Human Hair Follicle Dermal Papilla Cells and Hair Growth in C57BL/6 Mice by Modulating WNT/ $\beta-C a t e n i n$ and STAT Signaling. Biomol Ther (Seoul) 2016;24:572-80.

42. Yoshida Y, Soma T, Matsuzaki T, et al. Wnt activator CHIR99021-stimulated human dermal papilla spheroids contribute to hair follicle formation and production of reconstituted follicle-enriched human skin. Biochem Biophys Res Commun 2019;516:599-605.

43. Millar SE. Molecular mechanisms regulating hair follicle development. J Invest Dermatol 2002;118:216-25.

Cite this article as: Li Z, Zhang J, Li M, Tang L, Liu H. Concentrated nanofat: a modified fat extraction promotes hair growth in mice via the stem cells and extracellular matrix components interaction. Ann Transl Med 2020;8(18):1184. doi: 10.21037/atm-20-6086 